\title{
Can Desarda Technique Repair all Adult Inguinal Hernia?
}

\author{
Hama Y., \\ Department of Surgery,
}

Faculty of Health Sciences of Universite Abdou Moumouni

Department of Surgery of National Hospital of Niamey, Niger

Kadi I.,

Aichatou A. S.,

Adama S.,

Department of Surgery of National Hospital of Niamey, Niger

Yacouba H. D.,

Sani R.,

Department of Surgery,

Faculty of Health Sciences of UniversiteAbdou Moumouni

Department of Surgery of National Hospital of Niamey, Niger

Doi:10.19044/esj.2019.v15n24p102 URL:http://dx.doi.org/10.19044/esj.2019.v15n24p102

Abstract

Objectives: This paper focuses on reporting the results of the daily use of the Desarda technique for the treatment of adult inguinal hernias without selection of patients at national hospital of Niamey. Patients and Method: This was a prospective and descriptive study from December 2012 to August 2014. It included 76 patients aged over 15 years who underwent inguinal hernia repair by Desarda technique without any selection. The variables studied include the type of inguinal hernia, the Nyhus class of inguinal hernia, the operating time, the postoperative pain, and the hernia recurrences. Results: There were 74 men and 2 women. The average age of the patients was 43.6 years [ \pm 17.6$]$. Inguinal hernia was found in $57.9 \%$ of patients. There were $76.31 \%$ simple hernias, $15.80 \%$ recurrent hernias, $2.63 \%$ intractable hernias by loss of domain, and $5.26 \%$ strangulated hernias. $90.79 \%$ of the patients were operated under spinal anesthesia. The most frequent types of inguinal hernia were Nyhus type 2 found in $35.52 \%$ of patients, followed by Nyhus type $3 \mathrm{~b}$ found in $30.26 \%$ of patients. The mean operative time was 46.67 [ \pm 11.5] minutes. The immediate operative follow-up was marked by the occurrence of a hematoma of the scrotum in two patients. There was no recurrence of chronic postoperative pain after a median follow up of 40 
months. Conclusion: The Desarda technique could be a good alternative for the cure of all inguinal hernias of adults.

Keywords: Desarda technique, Inguinal hernia, Niamey National Hospital

\section{Introduction}

The inguinal hernia repair is one of the most common surgical procedures in adults (Lerut, 2000). The so-called "tension-free" prosthetic hernioplasty techniques have significantly reduced postoperative recurrences (Willaert, 2012; Nikkolo, 2016) but did not reduce the chronic postoperative pains (Lange, 2016). However, a new technique of aponeurroplasty described by Desarda based on the closure of the posterior wall of the inguinal canal by a flap of the fascia of the external oblique muscle would give promising results (Desarda, 2001). According to Dieng et al. this technique of "physiological repair" (Desarda, 2006) of the inguinal hernia deserves to be exploited in lowresource countries (Dieng, 2012). In fact, inguinal hernia repair is the most performed surgical procedure at the Niamey National Hospital in Niger (Harouna, 2000; Harouna, 2000). In this country, only a limited number of patients benefit from prosthetic hernia repair because prostheses are still not very accessible (Sani, 2004). Therefore, our study aims to report the general use of the Desarda technique for the treatment of adult inguinal hernias on a daily basis without selection of patients at Niamey National Hospital in Niger.

\section{Patients and Method}

This was a 21-month descriptive prospective study from December 2012 to August 2014. The study involved 76 patients over 15 years of age who underwent simple or complicated inguinal hernia repair with the Desarda technique by the same senior surgeon. After the diagnosis of inguinal hernia based on the clinical examination, the patients benefited from further routine investigations which were necessary for the safety of the surgical procedure. The operation was carried out by inguinal cutaneous incision. Then, the aponeurosis of the external oblique muscle was incised. After the hernial sac was dissected and resected in oblique hernias or buried in direct hernias, the medial leaf of the aponeurosis of the external oblique muscle was sutured to the inguinal ligament by separate points of $2 / 0$ silk. The sutured aponeurosis was then incised to define a fascial flap about $1.5 \mathrm{~cm}$ wide. The free edge of this fascial flap was sutured to the internal oblique muscle by separate points of 2/0 silk. The 2 free aponeurosis lefts were then sutured in front of the spermatic cord before suturing the subcutaneous and cutaneous tissues. The variables studied include the type of inguinal hernia, the Nyhus class of inguinal hernia, the operating time, the postoperative pain, and the hernia recurrences. 


\section{Results}

Seventy-six patients were included in the sample. They were 74 men and 2 women. The average age of the patients was 43.6 years [ \pm 17.6$]$ with extremes of 17 and 80 years. Manual labourers and farmers accounted for $36.83 \%$ of our patients. The inguinal hernia was found in $57.90 \%$ of patients. There were $76.31 \%$ of simple inguinal hernias (Figures 1, 2, and 3), 15.80\% of recurrent inguinal hernias, 5.26\% strangulated inguinal hernias, and $2.63 \%$ of intractable hernia by loss of the domain related to adhesion in hernia sac bag (Figure 4). Strangulated viscera were small bowel in 3 patients and omentum in 1 patient. However, there was no necrosis of the strangulated hernial contents in our patients. Locoregional anesthesia was used in $90.79 \%$ of patients and $9.21 \%$ of patients had general anesthesia. Inguinal hernia was classified as Type 1 of Nyhus in 5.26\% of patients, Nyhus type 2 in $35.52 \%$ of patients, Nyhus type $3 \mathrm{~A}$ and $3 \mathrm{~B}$ respectively in $13.16 \%$ and $30.26 \%$ of patients, and type 4 of Nyhus in $15.80 \%$ of patients. The average duration of the procedure was 47.67 [ \pm 11.52$]$ minutes with extremes of 30 to 75 minutes. The immediate operative follow-up was marked by the occurrence of a hematoma of the scrotum in 2 patients. They were treated by antiinflammatory drugs. One of these 2 patients had scrotal hematoma evacuation after failure of anti-inflammatory drugs treatment. All of our patients who underwent simple inguinal hernia repair had been released within 24 hours of surgery. The others had left the hospital the day after the surgery. Fifteen patients were lost to follow-up and 3 patients died. The median duration of the follow-up of the remaining 58 patients was 40 months. We did not notice any chronic postoperative pain among the 58 patients during the follow-up. However, there was one recurrence in our series after 37 months of follow up which occurred in a driver who was 47 years old.

\section{Discussion}

Inguinal hernia is still one of the most common surgical conditions in black Africa. In Niger, the inguinal hernia repair is at the forefront of all surgical procedures (Harouna, 2000; Sani, 2007). In this country, the most commonly used hernia repair techniques are herniorrhaphy techniques, essentially the modified Bassini's repair (Harouna, 2000; Harouna, 2000; Sani, 2007). Mesh hernia repairs are only performed in few teaching hospitals (Sani, 2004). The laparoscopic repairs are not done yet. Herniorrhaphies, the predominant techniques in Niger, use the only suture of the anatomical structures constituting the inguinal canal to repair the dehiscence of fascia transversalis. They are therefore providers of tension and recurrence hernia and are less used around the world for the benefit of techniques using so-called " tension-free " prostheses which currently constitute the gold standard (EU HerniaTrialists Collaboration, 2000). However, the prostheses are not widely 
used in our country because of their cost, but also for fear of infections of the operating site related to the conditions of practice. These conditions are common to many hospitals in sub-Saharan Africa such as: the crowded living conditions of patients in the hospital units, the poor state of the operating rooms, and the insufficiency of hospital linen. This makes each patient use what he has and often a sheet is used for several days. In this context, the physiological Desarda repair should be the most appropriate option to be used (Desarda, 2006). In fact, the Desarda technique would give results which are comparable to the Lichtenstein technique (ManyIlirah, 2012; Szopinski, 2012). Jones et al. reported about Desarda's technique that "besides being tension-free, it is simple to perform, requiring neither mesh nor extensive dissection" (Jones, 2015). Desarda's technique is feasible whether it is simple inguinal hernia or complicated inguinal hernia. Desarda et al. reported 16 cases of recurrent inguinal hernia, 3 cases of strangulated inguinal hernia, and 16 cases of inguinal hernia with loss of domain from a total of 229 patients (Desarda, 2006). In our series, $90.79 \%$ of patients were operated on spinal anesthesia. Although the Desarda technique is feasible under local anesthesia (Desarda, 2006). Our preference is for locoregional anesthesia because it confers a good operating comfort for the surgeon and good postoperative analgesia for the patient. Nevertheless, it can give a reversible urinary retention. Pain is an important criterion for patient satisfaction and assessment of different surgical techniques. In effect, all techniques of hernia surgery would be responsible for acute pain depending on the degree of dissection and tissue injury (Liem, 1997). In our study, acute postoperative pain was found in $30.27 \%$ of patients. This percentage of acute pain is comparable to the $34 \%$ of acute postoperative pain reported by Dieng et al. (Dieng, 2012). Chronic postoperative pain is a constant but variable complication of all hernia repair techniques (Cunningham, 1996). Cunningham et al. classified chronic postoperative pain into somatic pain (by stressing musculo-aponeurotic structures and triggered by exercise), nerve pain (by iliohypogastric nerve, ilioinguinal nerve, and genital branch of the genitofemoral nerve lesions, typically violent and brief), and visceral pain (by obstruction of the ductus deferens, by cicatricial fibrosis triggered by ejaculation) (Cunningham, 1996). However, we assume that regardless of the surgical technique, the identification and preservation of the nerves and avoiding to narrow the spermatic cord may reduce the incidence of chronic postoperative nerve pain and visceral pain. In addition, the Dersarda technique would give less fibrosis. Therefore, there will be less postoperative nerve sheathing and cord obstruction. Indeed, we did not notice any chronic postoperative pain in our series.

Hernial recurrence after inguinal hernia repair is one of the major challenges of all hernia repair techniques. It is a multifactorial complication 
depending on 1) The operated: the occurrence of a hernia recurrence is favored by the female, the elderly over 50 years, smoking, and heredity (Jansen, 2009; Zheng, 2002); 2) Type of hernia: direct inguinal hernias, sliding inguinal hernias, large inguinal hernias, and recurrent inguinal hernias are risk factors for hernia recurrence (Burcharth, 2014; International guidelines for groin hernia management, 2018); 3) The operator: the inexperience of the operator, and its volume of activity (International guidelines for groin hernia management, 2018; Gopal, 2013); and 4) The hernia repair technique used: tension-free techniques give less recurrence than herniorrhaphies. The Desarda technique will give recurrence rates which are similar to the Lichtenstein technique (EU HerniaTrialists Collaboration, 2000; Jones, 2015), unlike the latter. The Desarda technique does not interact with the nerve structures of the inguinal region and the ductus deferens.

\section{Conclusion}

The Desarda technique can be routinely used to repair simple inguinal hernias and complicated inguinal hernias with satisfactory results.

\section{Figures}

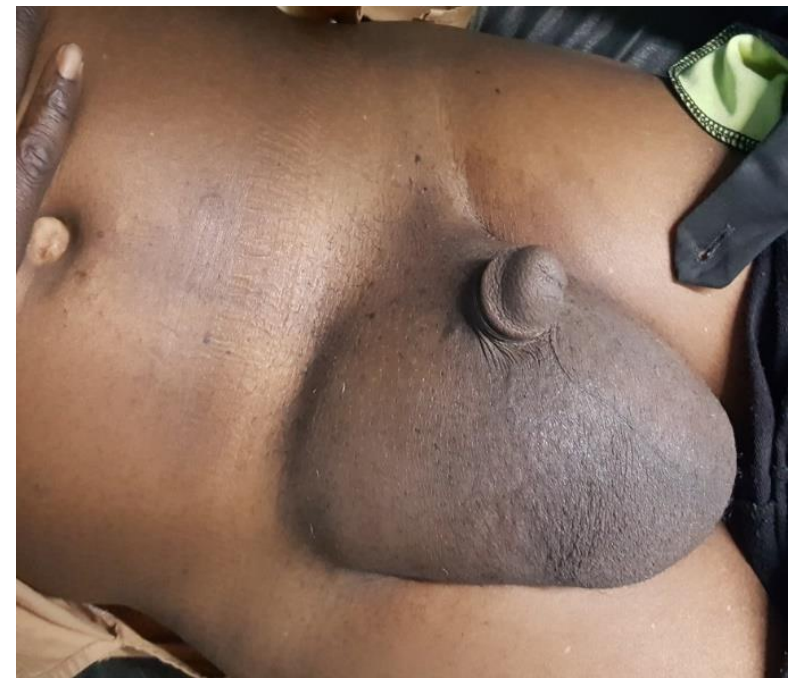

Figure1.Right inguinal hernia in a young adult 


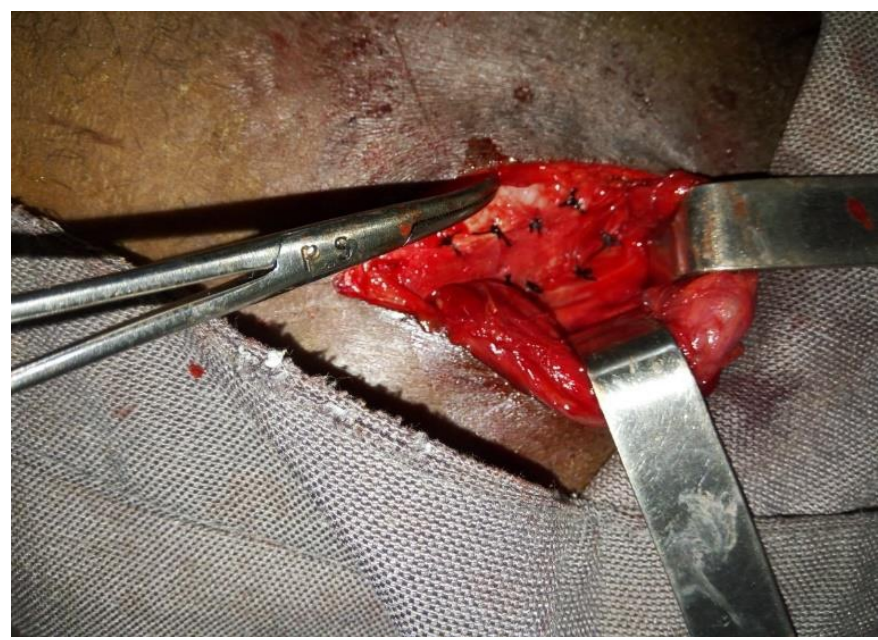

Figure2. Reinforcement of transversalis fascia by a flap of the fascia of the external oblique muscle between the 2 rows of separated points of $2 / 0$ silk

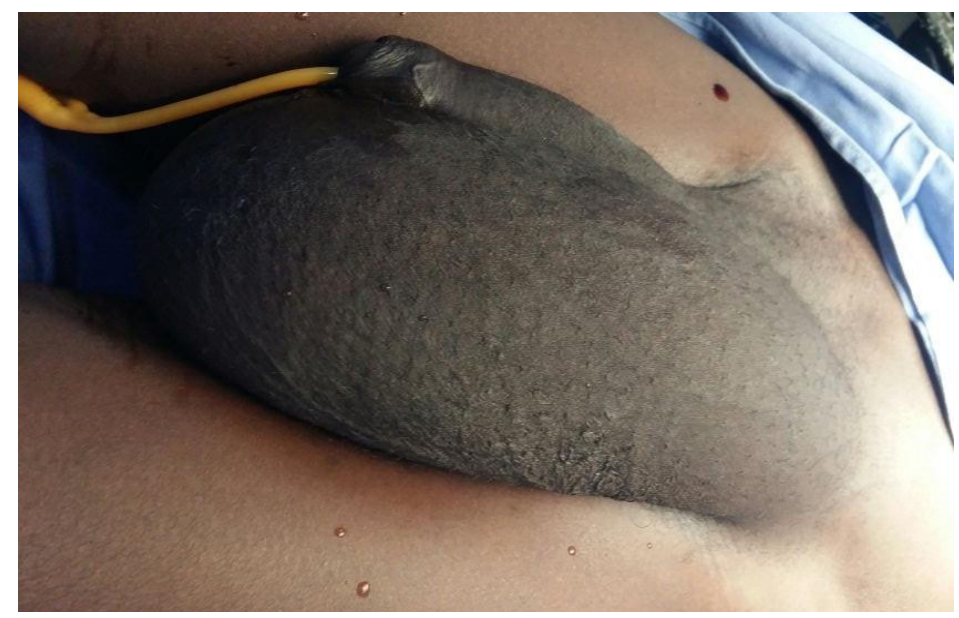

Figure3. Left inguinal hernia having loss of domain

\section{References:}

1. Burcharth, J. (2014). The epidemiology and risk factors for recurrence after inguinal hernia surgery. Dan Med J. mai; 61(5):B4846.

2. Cunningham, J., Temple, WJ., \& Mitchell P et al. (1996). Cooperative hernia study. Pain in the post repair patient. Ann Surg; 224(5) :598602

3. Dieng, M., Cissé, M., \& Seck, M. (2012). Cure des hernies inguinales simples de l'adulte par plastie avec l'aponévrose du grand oblique : technique de Desarda. E-memoires ANC; 11(2) : 69-74.

4. Desarda, MP. (2006). Physiological repair of inguinal hernia: a new technique (study of 860 patients). Hernia ; 10 :143-146. 
5. Desarda, MP. (2001). New method of inguinal hernia repair: a new solution. Anz J Surg; 71:241-4.

6. EU HerniaTrialists Collaboration (2000). Mesh compared with nonmesh methods of open groin hernia repair: systematic review of randomized controlled trials. Br J Surg; 87: 854-59

7. Gopal, SV. \& Warrier, A. (2013). Recurrence after groin hernia repairrevisited. Int J Surg. 1 juin;11(5):374-7

8. Harouna, YD. (2000). Le vécu de la pathologie herniaire par le chirurgien africain. l'exemple du Niger (Afrique). Med Afr Noire.; 47(7) : 353

9. Harouna, YD., Seibou, A., \& Manzo R et al. (2000). La hernie inguinale simple de l'adulte : étude médico-économique à propos e de 244 cas. Med Afr Noire;47(6) : 292-7

10. Harouna, YD. \& Le vécu de la pathologie herniaire par le chirurgien africain (200). l'exemple du Niger (Afrique). Med Afr Noire.; 47(7) : 353

11. International guidelines for groin hernia management (2018). Hernia. 1 févr;22(1):1-165.

12. Jones, P., Jones, S., \& Guarneri F et al. (2015). Topic: inguinal HerniaMesh vs non-mesh;19: S265-S266

13. Jansen, PL., Klinge, U., Jansen, M., \& Junge, K. (2009). Risk factors for early recurrence after inguinal hernia repair. BMC Surg. 9:18

14. Lerut, J., Ciccarelli, O., \& Astarci, P. (2000). La chirurgie herniaire. Louvain med. 2000;119:452-7.

15. Lange, JFM., Meyer, VM., \& Varopai DA et al. (2016). The role of surgical expertise with regard to chronicpostoperative inguinal pain (CPIP) after Lichtenstein correction of inguinal hernia: asystematicreview. Hernia; 1-8.

16. Liem, MS., Halsema, JA., \& Van der Graf Y et al. (1997). Cost effectiveness of extra peritoneal laparoscopic inguinal hernia repair: a randomized comparison with conventional herniorraphy. Coala trail group. Ann Surg; 226(6): 668-75.

17. ManyIlirah, W., Kijjambu, S., Upoki, A., \& Kiryabwire, J. (2012). Comparaison of non-mesh (Desarda) and mesh (Lichtenstein) methods for inguinal hernia repair among black African patients: a short-term double-blind RCT. Hernia; 16 :133-144

18. Nikkolo, C., Vaasna, T., \& Murruste M et al. (2016). Three-year results of a single-centre single-blinded randomised study evaluating the impact of mesh pore size on chronic pain after Lichtenstein hernioplasty. Scand j surg; 105(3): 141-6 
19. Szopinski, J., Dabrowiecki, S., \& Pierscinski S et al. (2012). Desarda Versus Lichtenstein technique for primary inguinal hernia treatment: 3-years results of randomised clinical trial. World J Surg; 36: 984-992 20. Sani, R., MC Gee, JM., \& Illo A et al. (2004). Cure de la hernie inguinale selon la technique «tension free» de Lichtenstein: expérience de l'hôpital national de Niamey à propos de 47 cas. J Afr Chir Digest; 4(2): 359-366.

21. Sani, R., Gbane, M., \& Hennaux M et al. (2007). Prise en charge des hernies inguinales simples : comparaison des aspects économiques et thérapeutiques d'un hôpital de district et l'hôpital national de NiameyNiger. Med Afr Noire; 54(4) :215-9

22. Willaert, W., De Bacquer, D., \& Rogiers X et al (2012). Open Preperitoneal Techniques versus Lichtenstein Repair for elective Inguinal Hernias (Review). Cochrane Database of Systematic Reviews, Issue 7. Art. No.: CD008034. DOI: 10.1002/14651858.CD008034.pub2

23. Zheng, H., Si, Z., Kasperk, R., Bhardwaj, RS., Schumpelick, V., \& Klinge $U$ et al. (2002). Recurrent inguinal hernia: disease of the collagen matrix? World J Surg; 26(4):401-8. 